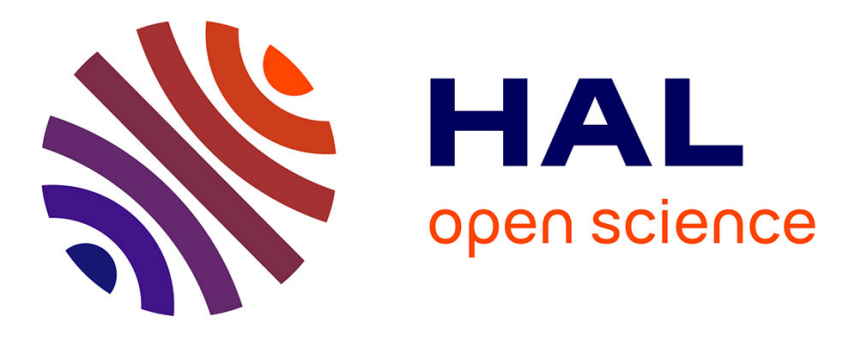

\title{
Grafted Nanoparticle Surface Wetting during Phase Separation in Polymer Nanocomposite Films
}

Shawn M Maguire, Michael J Boyle, Connor R Bilchak, John Derek Demaree, Austin W Keller, Nadia M Krook, Kohji Ohno, Cherie R Kagan, Patrice Rannou, Christopher B Murray, et al.

\section{To cite this version:}

Shawn M Maguire, Michael J Boyle, Connor R Bilchak, John Derek Demaree, Austin W Keller, et al.. Grafted Nanoparticle Surface Wetting during Phase Separation in Polymer Nanocomposite Films. ACS Applied Materials \& Interfaces, 2021, 13 (31), pp.37628-37637. 10.1021/acsami.1c09233 . hal03418118

\author{
HAL Id: hal-03418118 \\ https://hal.science/hal-03418118
}

Submitted on 6 Nov 2021

HAL is a multi-disciplinary open access archive for the deposit and dissemination of scientific research documents, whether they are published or not. The documents may come from teaching and research institutions in France or abroad, or from public or private research centers.
L'archive ouverte pluridisciplinaire HAL, est destinée au dépôt et à la diffusion de documents scientifiques de niveau recherche, publiés ou non, émanant des établissements d'enseignement et de recherche français ou étrangers, des laboratoires publics ou privés. 


\section{Grafted Nanoparticle Surface Wetting during Phase Separation in Polymer Nanocomposite Films}

Shawn M. Maguire, Michael J. Boyle, Connor R. Bilchak, John Derek Demaree, Austin W. Keller, Nadia M. Krook, Kohji Ohno, Cherie R. Kagan, Christopher B. Murray, Patrice Rannou, and Russell J. Composto*

ABSTRACT: Wetting of polymer-grafted nanoparticles (NPS) in a polymer nanocomposite (PNC) film is driven by a difference in surface energy between components as well as bulk thermodynamics, namely, the value of the interaction parameter, $\mathrm{X}$. The interplay between these contributions is investigated in a PNC containing $25 \mathrm{wt} \%$ polymethyl methacrylate (PMMA)- grafted silica NPs (PMMA-NPs) in poly(styrene-ran-acrylonitrile) (SAN) upon annealing above the lower critical solution temperature (LCST, $\left.160{ }^{\circ} \mathrm{C}\right)$. Atomic force microscopy $(\mathrm{AFM})$ studies show that the areal density of particles increases rapidly and then approaches $80 \%$ of that expected for random close-packed hard spheres. A slightly greater areal density is observed at $190^{\circ} \mathrm{C}$ compared to $170{ }^{\circ} \mathrm{C}$. The PMMA-NPs are also shown to prevent dewetting of PNC films under conditions where the

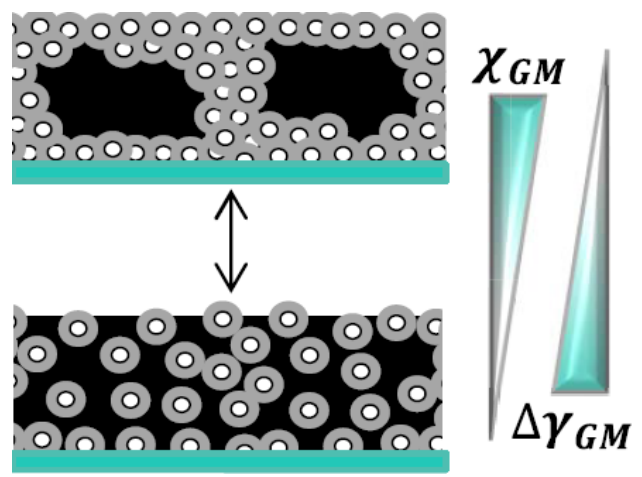
analogous polymer blend is unstable. Transmission electron microscopy (TEM) imaging shows that PMMANPs symmetrically wet both interfaces and form columns that span the free surface and substrate interface. Using grazingincidence Rutherford backscattering spectrometry (GI-RBS), the PMMA-NP surface excess ( $\left.Z^{*}\right)$ initially increases rapidly with time and then approaches a constant value at longer times. Consistent with the areal density, $Z^{*}$ is slightly greater at deeper quench depths, which is attributed to the more unfavorable interactions between the PMMA brush and SAN segments. The $Z^{*}$ values at early times are used to determine the PMMA-NP diffusion coefficients, which are significantly larger than theoretical predictions. These studies provide insights into the interplay between wetting and phase separation in PNCs and can be utilized in nanotechnology applications where surface-dependent properties, such as wettability, durability, and friction, are important.

KEYWORDS: polymer surfaces, polymer nanocomposites, surface segregation, grafted nanoparticles, diffusion, wetting

\section{INTRODUCTION}

Polymer nanocomposites (PNCs), materials that are composed of nanoscale organic or inorganic fillers in a polymer matrix, are of great scientific interest because they can be designed with many combinations of components to create functional materials with vastly different properties.1-3 There are many approaches to control and tailor the structure and properties of PNCs, including adding bare or grafted nanoparticles (NPs) to a polymer matrix for enhanced mechanical properties,4-8 employing mobile nanoparticle (NP) systems to build hierarchical functional composite structures, 9 and using NPs to impose extreme nanoconfinement on polymer chains, resulting in composites with improved thermal stability and drastic reductions in polymer segmental dynamics.10-12 Because the synergistic properties of PNCs are directly correlated with the spatial arrangement of the inorganic NPs within the polymer host,13-17 different strategies for controlling the dispersion of inorganic fillers have been investigated. Whereas a homogeneous distribution of NPs is sometimes desired, some properties benefit from directing NP assembly at surfaces and in the bulk. For instance, wettability, durability, and friction can be controlled by preferential localization of NPs to interfaces.

Previously, the surface composition of a miscible polymer blend has been shown to differ from the bulk composition because of the difference in surface energies and thermodynamic interactions between polymers.18-21 For immiscible polymer blends undergoing phase separation, wetting of the lower surface energy component at the surface initially dominates the morphological evolution.22 As a result, unique polymer blend morphologies can be generated by balancing the degree of phase separation and wetting within the films. For example, films of deuterated poly(methyl methacrylate) (dPMMA) and poly(styrene-ran-acrylonitrile) (SAN) undergo different stages of phase evolution and wetting, including the formation of a bicontinuous morphology, followed by discrete dPMMA domains, sandwiched between dPMMA wetting layers.23-28 Here, symmetric wetting occurs due to the preferential attraction of dPMMA to the surface and oxide substrate interface.29 In binary polymer blends, these studies demonstrated that tuning the interplay between phase separation and wetting led to unique morphologies, many of which cannot be readily produced using traditional nanotechnology processing methods. These studies were then expanded to include phase-separating ternary polymer/ polymer/NP mixtures, that is, bigels, where the interface between polymer domains becomes decorated with NPs to arrest the growth of bicontinuous structures.24,26,28,30-32

In this work, we mix PMMA-grafted silica NPs (PMMANPs) and SAN, follow the thin-film morphological evolution during phase separation, and compare results to the analogous thin-film homopolymer blends, namely, PMMA/SAN. By transmission electron microscopy (TEM), we identify three phase morphologies. Upon spin-coating and drying, the NPs in the PNC are homogeneous, consistent with a thermodynamically stable, well-defined initial state. During the early stages of phase separation upon annealing, PMMA-NPs begin to wet both the free surface and substrate as well as aggregate in the bulk. Akin to dPMMA, PMMA-NPs wet both the air and oxide interfaces because of their preferential attraction. At longer durations of time, a trilayer structure, PMMA-NP-rich/SAN-rich/ PMMA-NP-rich, is formed parallel to the substrate. Specifically, columns of PMMA-NP aggregates span the two wetting layers and are surrounded by the SAN-rich phase. A similar morphology was observed for the PMMA/SAN blend with PMMA domains spanning the PMMA wetting layers. To quantify the PMMA-NP concentration at the surface, the number 
density and surface excess $\left(Z^{*}\right)$ of PMMA-NPs were measured by atomic force microscopy (AFM) and grazing incidence Rutherford backscattering spectrometry (GI-RBS). The number density increases rapidly and then approaches a plateau value, ca. $80 \%$ of the random close packing value. Similarly, $Z^{*}$ increases rapidly and approaches a constant value, consistent with a diffusion-limited process that depends on the quench depth. From the $Z^{*}$ values at early times, the diffusion coefficients of PMMA-NP are determined and found to be much larger than theoretical predictions.

These studies provide insights into the interplay between wetting and phase separation in PNCs and can be utilized in nanotechnology applications where surface-dependent properties are important. Additionally, the replacement of PMMA with PMMA-NPs introduces several advantages compared to the pure blend case. First, the nanocomposite films can be used as templates for aligning and controlling the dispersion of NPs within a polymer host. Second, the silica-rich domains will etch much slower than the SAN-rich phases, which allows for the fabrication of porous membranes that could be used in the as prepared morphology, backfilled with an inorganic material, or grafted with a polymer brush. Third, unlike the analogous polymer blend, the PNC film is stable and does not dewet from the substrate during late-stage phase separation. This stability is attributed to NP jamming, which prevents the development of long-range interfacial fluctuations that can lead to film rupture. Finally, we note that the mechanical properties of the nanocomposite should be improved, relative to the polymer blend, because the jammed NP wetting layers and NP columns should resist deformation, similar to the suppression of the interfacial fluctuations. Details will be presented in a future study.

\section{RESULTS AND DISCUSSION}

The bulk phase diagrams of a PMMA (91 kg/mol)/SAN $(118 \mathrm{~kg} / \mathrm{mol})$ blend and of a PMMA $(19 \mathrm{~kg} / \mathrm{mol})-\mathrm{NP} / \mathrm{SAN}(118$ $\mathrm{kg} / \mathrm{mol}$ ) PNC have been previously constructed using complementary techniques of cloud point, TEM, and small angle X-ray scattering.33,34 Figure 1a depicts the phase diagram of the polymer blend, PMMA/SAN, which exhibits a lower critical solution temperature (LCST) of $160^{\circ} \mathrm{C} .33$ The phase diagram of the PNC, PMMA-NP/SAN, is plotted as a function of PMMA-NP weight fraction in Figure 1b.34 Comparing Figures 1a,b shows that the phase boundaries for the PNC and the binary polymer blends are similar near the critical point with a shift toward lower PMMA (PMMANP) weight fractions. Namely, the miscibility of SAN in PMMA-NP increases, whereas the miscibility of PMMA-NP in SAN changes only slightly compared to the pure blend case. If Figure $1 \mathrm{~b}$ was plotted against only the PMMA weight fraction, the boundary shift toward lower PMMA concentration would be further enhanced. Additionally, Figure 1b shows that the binodal width decreases and the critical composition shifts to lower weight fractions of PMMA-NP compared to those shown in Figure 1a. We attribute this increased miscibility window to a decrease in the PMMA molecular weight, namely, decreasing from 91 to $19 \mathrm{~kg} / \mathrm{mol}$. An analysis of this behavior was described along with a comparison with the PRISM theory in the work by Maguire et al.34 Overall, this result indicates that dense polymer brushes grafted to NPs $\left(0.7\right.$ chains $\left./ \mathrm{nm}^{2}\right)$ exhibit enthalpic interactions comparable to their analogues in a polymer blend. The PMMA-NP/SAN system is an ideal candidate for studying the interplay between wetting and phase separation for several reasons. First, the bulk phase diagram (Figure 1b) follows the LCST behavior, allowing for the preparation of thermodynamically stable mixtures at room temperature. This is in contrast to an upper critical solution temperature (UCST) PNC where the prepared films will be kinetically trapped and in a metastable state because of

a.

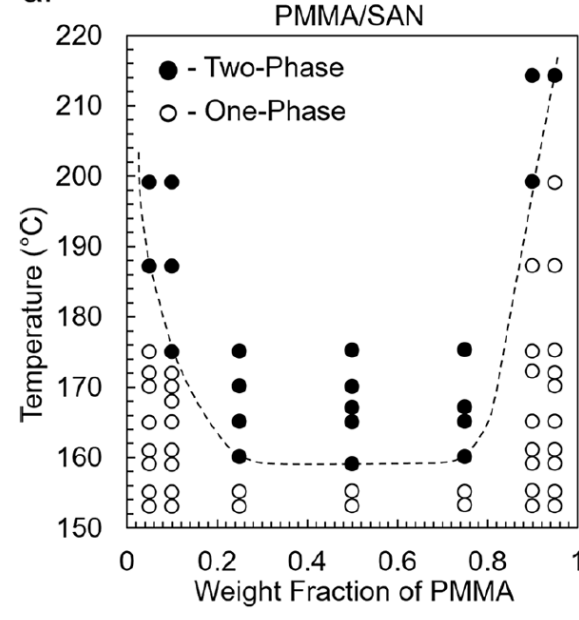

C.

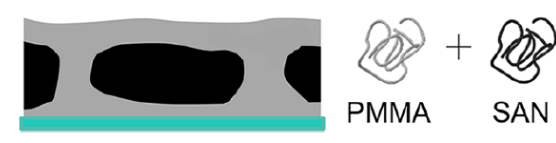

b.

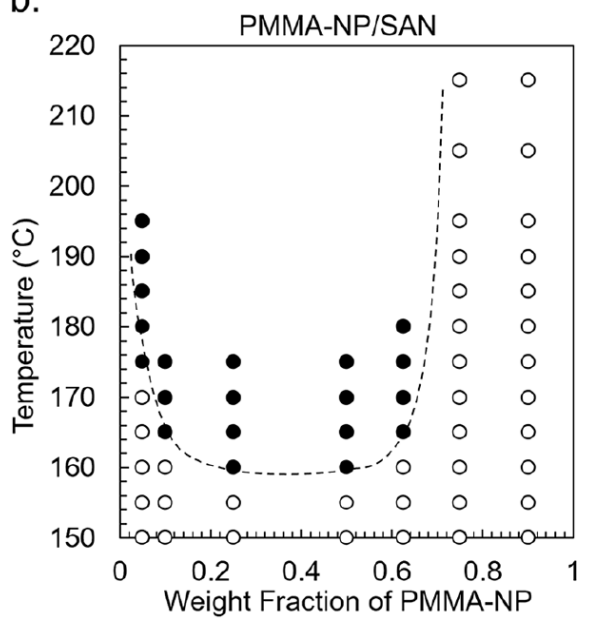

d.

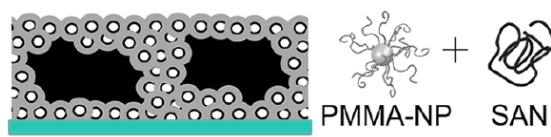

Figure 1. Comparison of the bulk phase diagrams of the polymer blend, PMMA $(91 \mathrm{~kg} / \mathrm{mol}) / \mathrm{SAN}(118 \mathrm{~kg} / \mathrm{mol})$, with the PNC, and PMMA (19 kg/mol)-NP/SAN (118 kg/mol). (a) Phase diagram of PMMA/SAN, which exhibits LCST behavior with a critical temperature of ca. $160^{\circ} \mathrm{C}$. (b) Phase diagram of a PMMA-NP/SAN where the molecular weight of SAN is the same as that in (a). The filled circles represent two-phase regions, whereas the open circles represent one-phase regions where NPs are dispersed. (c, d) Cartoon representations of the cross-sectional view of the thin-film morphologies in the two-phase region. The dashed lines are a guide to the eye. Parts of this image were adopted with permission from previous studies.32,33 
the processing temperature being significantly lower than most polymer Tg's. Furthermore, the critical temperature of ca. $160{ }^{\circ} \mathrm{C}$ is well above the glass transition temperature of the $25 / 75 \mathrm{wt} \%$ blend (ca. $120{ }^{\circ} \mathrm{C}$ ). Thus, we can investigate the effect of weak and strong thermodynamic driving forces, namely, shallow $\left(170{ }^{\circ} \mathrm{C}\right)$ and deep $\left(190{ }^{\circ} \mathrm{C}\right)$ quench depths, on simultaneous wetting and phase separation. Second, previous studies of phase separation in polymer blend thin films (i.e., PMMA/SAN) have shown that the PMMA-rich phase symmetrically wets both the free surface and silicon substrate upon demixing because of a large difference in surface energies between PMMA and SAN as well as the preferential attraction of PMMA to the oxide substrate.33 Because the silica particles are densely grafted with PMMA and PMMA-NP/SAN blends exhibit a comparable phase diagram to PMMA/SAN blends, these studies investigate whether PMMA-NPs also wet the free surface and silicon substrate during phase separation, similar to PMMA in the polymer blend. By monitoring the evolution of the PMMANP wetting layers, the governing kinetics of this PNC can be determined. Thus, these studies provide an alternative route toward measuring the diffusion coefficient of NPs.

To investigate wetting and phase separation, we characterized the PMMA-NP/SAN surface morphology by AFM, the bulk morphology by TEM, and the PMMA-NP surface excess by GI-RBS. To investigate the effect of thermodynamic driving forces on dynamics, the kinetics of wetting and phase separation were determined at 170 and $190{ }^{\circ} \mathrm{C}$, corresponding to 10 and $30^{\circ} \mathrm{C}$ quenches above the LCST. The inset of Figure 1c shows a representative cartoon of the PMMA/SAN thin-film cross-section corresponding to the morphology observed at a blend composition of ca. 25 wt \% PMMA during the intermediate stage of phase separation. Compared to bulk films, phase-separating, thin-film polymer blends undergo distinct evolutionary stages because of confinement.27 Therefore, it is important to note that this morphological evolution is only achieved under specific film thickness conditions and loadings of PMMA, one of which is denoted herein. As shown in a morphology map derived by Chung and co-workers, variations in film thickness (i.e., 50-1000 nm) and PMMA volume fraction (i.e., 0.3-0.8) can lead to six distinct pattern development regimes.27 Figure 1d represents the anticipated morphology of PMMA-NP/SAN when wetting and phase separation proceeds similarly for the PNC containing PMMA-NP of similar loading and film thickness. Namely, the PNC morphology would exhibit PMMA-NPs wetting both the free surface and silicon substrate with a middle region corresponding to the PMMA-NP domains (gray) separated by a continuous SAN-rich phase (black).

To probe the surface enrichment of PMMA-NPs during phase separation, AFM was used to quantify the particle number density at the surface as a function of annealing temperature and time. Figure 2a shows representative AFM images of the PNC surface with the height scale to the right of each image. The as-cast PNC (top left) shows uniformly dispersed PMMA-NPs with an average center-to-center interparticle distance of $55 \mathrm{~nm}$ based on the peak in the images' autocorrelation function (Figure $2 \mathrm{~b}$ ). A root-mean square roughness $\left(R_{\mathrm{q}}\right)$ of $\sim 2.0 \mathrm{~nm}$ is observed. Upon

a.

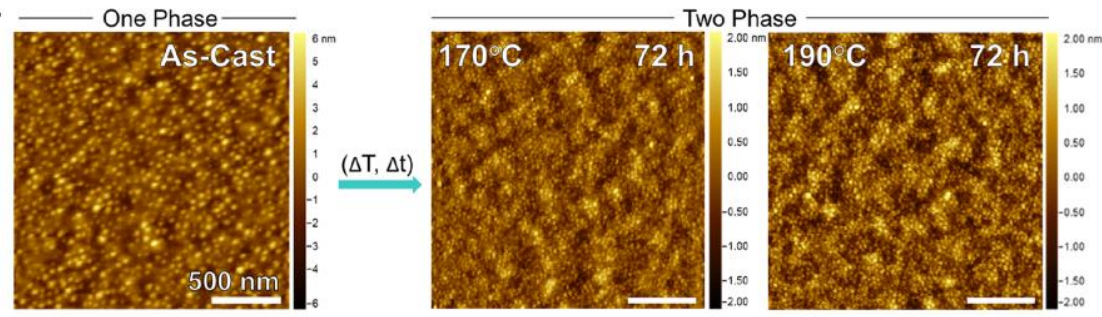

b.

c.
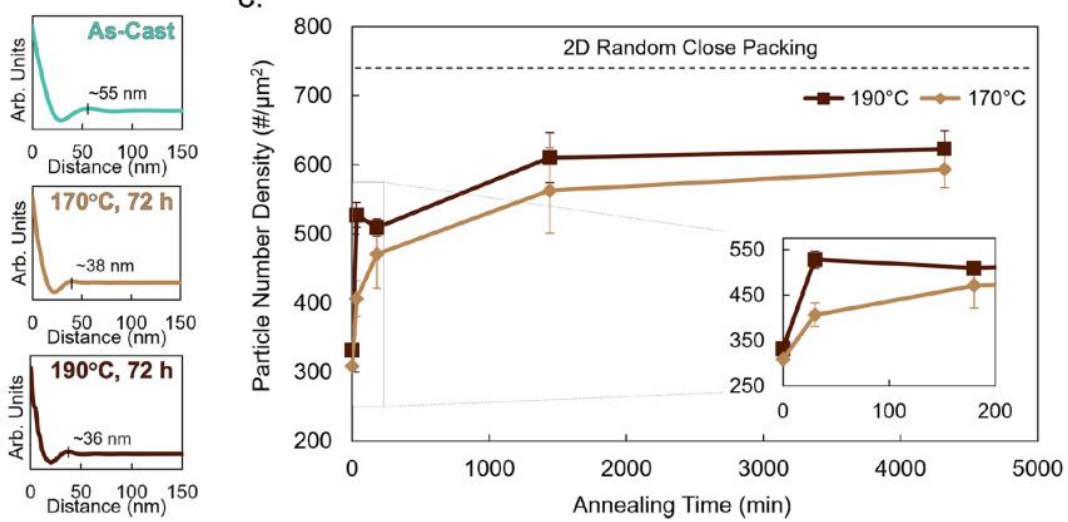

Figure 2. Surface morphology evolution of 25/75 wt \% PMMA-NP/SAN. (a) AFM height images of as-cast (top left) and annealed films at $170{ }^{\circ} \mathrm{C}$ (top middle) and $190^{\circ} \mathrm{C}$ (top right) after $72 \mathrm{~h}$. Scale bars are $500 \mathrm{~nm}$. (b) Autocorrelation functions of the AFM images, revealing lower values of interparticle separation upon annealing. (c) Increase in the NP number density upon annealing at 170 and $190^{\circ} \mathrm{C}$. The dashed line is the theoretical maximum number density of particles assuming random close packing of hard spheres with the same diameter as PMMA-NP.

annealing in the two-phase region for $72 \mathrm{~h}$, the number density of NPs at the free surface increases, accompanied by the formation of long-wavelength fluctuations in the film topography. The autocorrelation functions of the AFM images for the samples annealed at $170{ }^{\circ} \mathrm{C}$ (top middle) and $190{ }^{\circ} \mathrm{C}$ (top right) display peaks at 38 and $36 \mathrm{~nm}$, respectively. Compared to the one-phase sample, NPs pack more closely, consistent with the wetting of PMMA-NPs at the surface. Upon increasing the quench depth by $20^{\circ} \mathrm{C}$, the number density increases only slightly, suggesting that NPs are already well packed at $170{ }^{\circ} \mathrm{C}$. A complementary analysis method was used to determine NP spacing. For samples before and after annealing at 170 and $190^{\circ} \mathrm{C}$, Figure S2 shows AFM line profiles passing across the 
centers of the grafted NPs, where each maximum corresponds to the center of each NP. The center-to-center distances obtained directly from line profiles for each sample are in good agreement with the value determined by the respective autocorrelation function (Figure $2 b)$.

For the films annealed at 170 and $190^{\circ} \mathrm{C}$, the roughness, $\mathrm{R}_{\mathrm{q}}$, was determined to be ca. $0.6 \mathrm{~nm}$, indicating a smoother surface morphology compared to the one-phase region. By comparison, PMMA/SAN blend films annealed for the same amount of time at comparable quench depths display two orders of magnitude higher $\mathrm{R}_{\mathrm{q}}$ values than the PNC. This significantly higher roughness is attributed to capillary fluctuations in the blend film, which leads to macroscopic roughening of the surface and eventually film rupture.33 Interestingly, the low $R_{q}$ values in this study suggest that the wetting of the PMMA-NPs at the free surface results in a jammed morphology, preventing the formation of largeamplitude capillary fluctuations that would break the film. Therefore, these studies suggest that the wetting of NPs prevents film dewetting and increases thermal stability.

While the AFM images indicate an increase in NP packing, further image analyses have been performed to quantify the particle number density on the surface as a function of annealing temperature and time (see the Supporting Information for methods). For PMMA-NP/SAN films annealed at 170 and $190{ }^{\circ} \mathrm{C}$, Figure $2 \mathrm{c}$ shows the NP number density at the surface for annealing times up to 3 days. For both temperatures, the number density increases rapidly at short durations of time and then approaches a nearly constant value at longer durations (3 days). For comparison, the as-cast PNC films have an average number density of particles of $320^{ \pm 22} \mathrm{NP} / \mu \mathrm{m}^{2}$. This value is expected to be higher than that for a perfectly smooth surface because of the measured surface roughness, which allows for a greater number density of NPs because of an increase in the surface area. At the longest time (i.e., $72 \mathrm{~h}$ ), the number density of NPs at the surface increases to $593^{ \pm 26} \mathrm{NP} / \mu \mathrm{m}^{2}$ and $620^{ \pm 25} \mathrm{NP} / \mu \mathrm{m}^{2}$ at 170 and $190^{\circ} \mathrm{C}$, respectively, which is consistent with a closer packing of NPs determined by the autocorrelation function analysis shown in Figure $2 \mathrm{~b}$.

At long annealing times, the surface becomes saturated with NPs, and the number density approaches a constant value, as shown in Figure $2 \mathrm{c}$. To compare these values to the theoretical maximum number density of particles at a smooth surface, the two-dimensional (2D) random close packing of hard spheres with the same radii as the polymergrafted NPs $(19 \mathrm{~nm}$ ) is calculated and plotted in Figure 2c (dashed line). While the experimental data may slightly increase at longer times, the results show that the experimental measurements at 170 and $190{ }^{\circ} \mathrm{C}$ fall slightly below the theoretical maximum number density for the packing of pure randomly close-packed NPs by 20 and $16 \%$, respectively. The lower experimental number density may be attributed to (1) the limited ability of AFM to distinguish between tightly packed particles when the center-to-center distance is comparable in length to the tip diameter, (2) long-wavelength height fluctuations at the surface, resulting in indistinguishable particles in the minima, and (3) NP jamming at the interface that arrests further growth of the surface layer.

As observed by AFM, the PNC morphologies appear to be thermally stable (i.e., no dewetting) for annealing times up to 3 days, which is evident from the low values of the RMS roughness. In contrast to the PMMA/SAN blends where films dewet during the late-stage evolution of phase separation, the interfacial jamming of the PMMA-NPs in PMMANP/SAN blends arrests the morphology. Specifically, NP jamming dampens interfacial roughening, which is the main contribution to film rupture, and therefore increases film stability. Rupture and dewetting in polymer blends have been demonstrated extensively in previous studies.29,35,36 To experimentally probe the enhanced thermal stability of these films attributed to the interfacial jamming of the PMMA-NPs, optical microscopy (OM) images were taken. Figure 3 shows the representative OM images of a 25/75 wt \% PMMA-NP/ SAN film before and after annealing at $190{ }^{\circ} \mathrm{C}$ for 3 and $72 \mathrm{~h}$ relative to the PMMA/SAN analogue. Film thickness values are ca. $400 \mathrm{~nm}$ for both films. For both the PNC and polymer blend films, as-cast samples exhibit a uniform morphology. After 3 and $72 \mathrm{~h}$, the PNC film remains stable, with a homogeneous surface morphology (Figure 3a). Conversely, Figure $3 \mathrm{~b}$ demonstrates that the homopolymer blend is unstable after $72 \mathrm{~h}$ of annealing, with clear dewetting of the film. Note that these holes are surrounded by rims where polymer has been collected upon dewetting. These OM images support the hypothesis that the interfacially jammed PMMA NPs halt the formation of long-wavelength fluctuations at the surface and suppress film rupture.

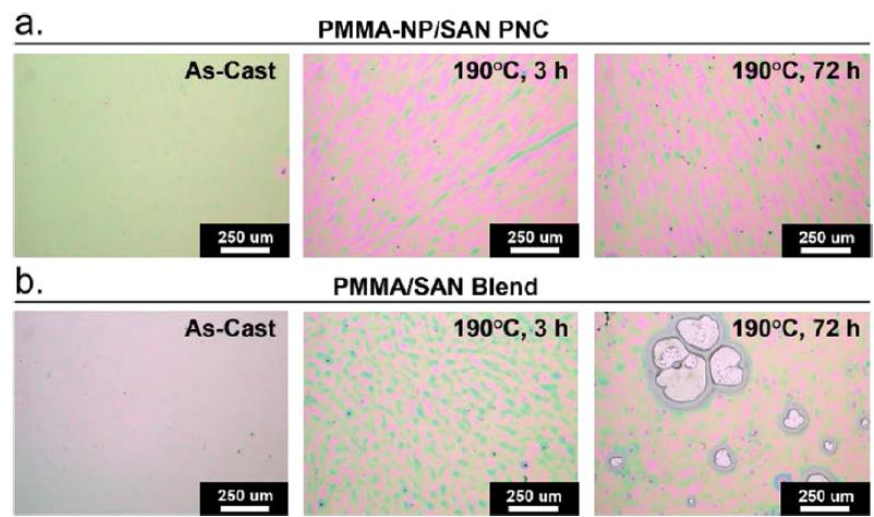

Figure 3. Optical microscopy images of (a) 25/75 wt \% PMMA-NP/ SAN and (b) 25/75 wt \% PMMA/SAN films annealed at $190{ }^{\circ} \mathrm{C}$ for up to $72 \mathrm{~h}$, respectively, using a $10 \times$ objective lens and $1.67 \times$ relay lens.

To track the internal phase evolution during PMMA-NP wetting, cross-sectional TEM images were taken for as-cast and annealed samples. Figures $4 a, b$ are representative images of 25/75 wt \% PMMA-NP/SAN films annealed at 170 ${ }^{\circ} \mathrm{C}$ (top row) and $190^{\circ} \mathrm{C}$ (bottom row), respectively, during different stages of phase evolution. Importantly, the as- 
cast samples exhibit a homogeneous dispersion of the PMMA-NPs within the SAN matrix (left column). Here, the favorable enthalpic interactions between the PMMA brush and the SAN matrix drive the initial dispersion of the NPs.34 However, when quenched into the two-phase region, PMMA-NPs wet the surface and substrate while undergoing phase separation with SAN in the bulk. After $3 \mathrm{~h}$ at $190^{\circ} \mathrm{C}$, a near monolayer of grafted NPs decorates the free surface of the film (Figure $4 \mathrm{~b}$, middle), whereas composites annealed at $170{ }^{\circ} \mathrm{C}$ (Figure $4 \mathrm{a}$, middle) exhibit a slightly lower surface coverage of PMMANPs. At $170^{\circ} \mathrm{C}$ for $3 \mathrm{~h}$, the bulk morphology shows elongated aggregates that are randomly oriented; however, the corresponding morphology at $190{ }^{\circ} \mathrm{C}$ begins to display aggregates

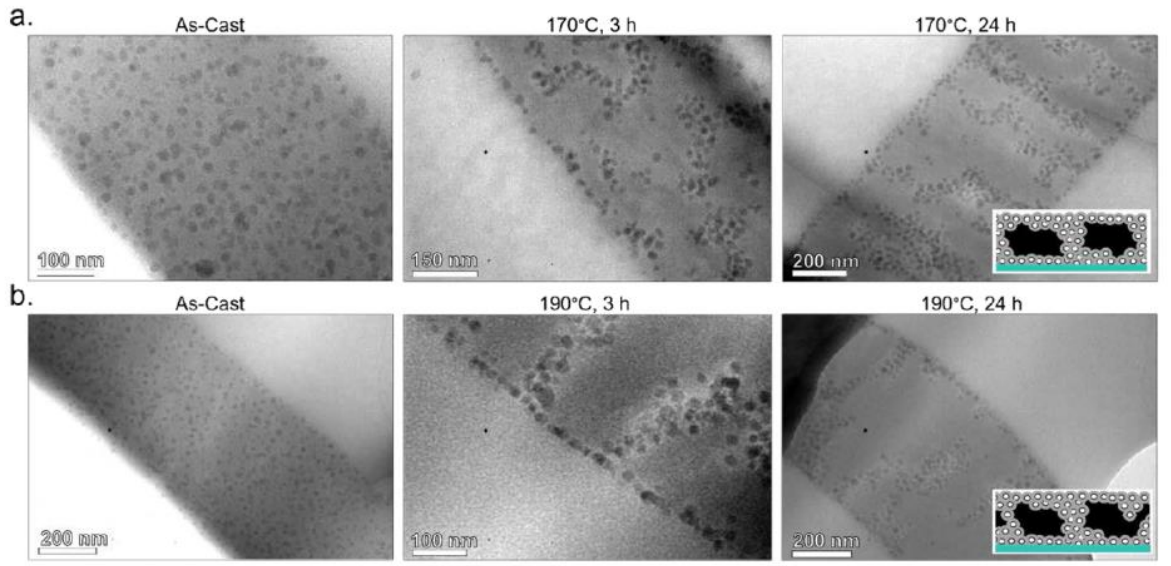

Figure 4. TEM cross-sectional images of $25 / 75$ wt \% PMMA-NP/SAN films annealed at $170{ }^{\circ} \mathrm{C}$ (a) and $190{ }^{\circ} \mathrm{C}$ (b) for 0,3 , and 24 h. Samples annealed for $24 \mathrm{~h}$ display internal morphologies represented by the cartoons in this inset and in Figure $1 \mathrm{~d}$.

perpendicular to the surface. For much thicker samples, small-angle X-ray scattering and TEM characterization show that discrete, elongated aggregates are formed that do not span the wetting layers.34 After $24 \mathrm{~h}$ (Figure $4 \mathrm{a}, \mathrm{b}$, right), the polymer composites assemble into a trilayer structure with layers of PMMA-NP-rich/SAN-rich/PMMANP-rich phases forming parallel to the substrate. PMMA/SAN films undergoing phase evolution and wetting exhibit a strikingly similar behavior. Specifically, during the intermediate stage of phase separation, PMMA domains form discrete columns that span the thickness of the film and then grow within the continuous SAN-rich matrix, which is sandwiched between the PMMA wetting layers at the free surface and substrate. Similar to the PMMA/SAN case, Figures 4a,b (right column) show that the SAN-rich phase surrounds the domains of PMMA-NPs that span the free surface and substrate (see inset schematics). However, one significant difference between the polymer blend and PNC is that the phase morphology continues to evolve in the former system, resulting in eventual film dewetting. This is in contrast to what is observed in the PNC where the jammed NPs stabilize the PNC.

To complement TEM and AFM studies, GI-RBS was used to measure the surface concentration profiles of PMMANP as a function of time. Figure 5 shows the silicon concentration (counts) as a function of depth for $25 / 75$ wt $\%$ PMMA-NP/SAN films (ca. $400 \mathrm{~nm}$ ) at 170 and $190^{\circ} \mathrm{C}$. The as-cast film (Figure $5 a$ ) exhibits a slightly increasing number of counts as a function of depth, consistent with an increase in the scattering cross-section for a homogeneous distribution of silicon from the $\mathrm{SiO}_{2} \mathrm{NPS}$. The increase in counts near $400 \mathrm{~nm}$ corresponds to scattering from the silicon substrate. This upturn obscures the backscattered silicon counts from silica NPs that have segregated to the substrate (cf. Figure 4). After annealing at $170^{\circ} \mathrm{C}$ (Figure 5a), the surface becomes enriched with PMMA-NP, corresponding to the appearance of the surface peak. To conserve silica NPs near the surface, this surface enrichment layer is adjacent to a depletion zone and causes the reduction in counts for samples annealed for 15 min and longer. As the PMMA-NP surface excess increases with time, the depth and width of the depletion region within the film also increase. At long durations of time, the surface excess stops growing, and the depletion zone weakens as the equilibrium between the surface and bulk concentration approaches. The profile below the depletion layer reflects the aggregation of NPs. The spectra for 25/75 wt \% PMMA-NP/ SAN films annealed deeper in the two-phase region, $190^{\circ} \mathrm{C}$, are shown in Figure $5 \mathrm{~b}$. Similar to the shallow quench depth case, the surface excess of PMMA-NPs monotonically increases as time increases. Qualitatively, the surface peak for the deeper quench depth (higher temperature) evolves faster than the evolution at a lower temperature (e.g., consistent with the AFM and TEM results shown in Figures 2 and 4, respectively).
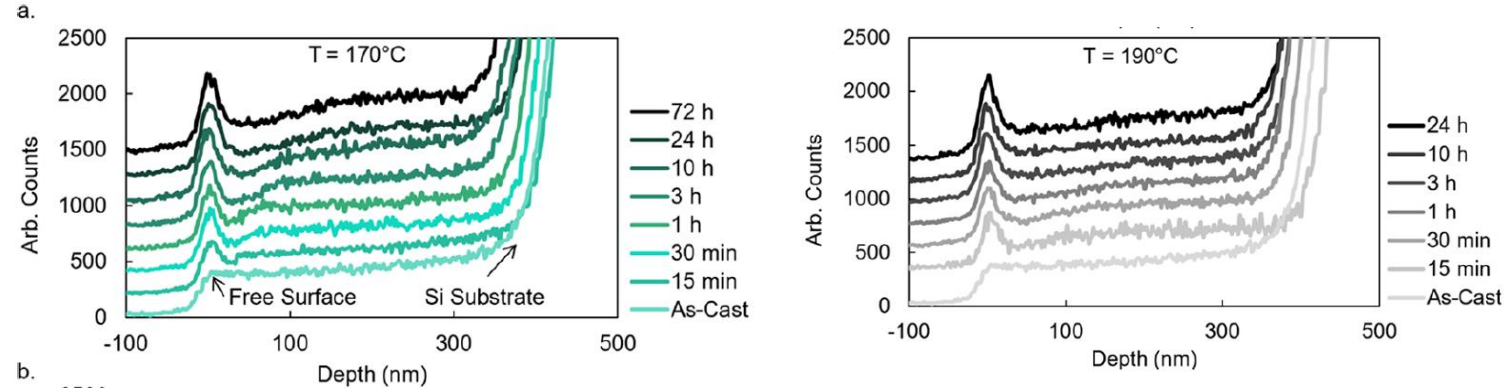

Figure 5. GI-RBS spectra of the silicon concentration depth profile in 25/75 wt \% PMMA-NP/SAN films (ca. $400 \mathrm{~nm}$ ) are shown at $170^{\circ} \mathrm{C}$ (a) and $190^{\circ} \mathrm{C}$ (b) as a function of annealing time. Spectra are shifted in counts for clarity. The surface peak near $0 \mathrm{~nm}$ represents an excess of PMMA-NP, whereas the upturn in counts near $400 \mathrm{~nm}$ is due to the silicon substrate. 
Using the depth profiles of PMMA-NPs, the diffusion coefficients of the grafted NPs were determined at 170 and $190^{\circ} \mathrm{C}$ and compared to analytical expressions derived previously for homopolymer blends.19,20 Figure 6 shows select depth profiles from PMMA-NP/SAN before and after annealing at $170{ }^{\circ} \mathrm{C}$ for $30 \mathrm{~min}$. Before determining $Z^{*}$ of PMMA-NPs (shaded area in Figure 6b), the as-cast profile is fit to a Gaussian function convoluted with a step function to extract the instrumental resolution (full width at half maximum, FWHM, of ca. $30 \mathrm{~nm}$ ). Next, the Gaussian instrumental resolution is convoluted with a solution to Fick's second law using appropriate boundary conditions:

$$
\varphi(x)=\frac{\varphi_{\text {Bulk }}}{2}\left[1+\operatorname{erf}\left(\frac{x-x_{0}}{4 \sqrt{D t}+2 \sigma^{2}}\right)\right]+\text { offset }
$$

where $\varphi_{\text {Bulk }}$ is the bulk composition of the film, $\sigma$ is the standard deviation of the instrumental resolution, $x_{0}$ is the step position, $t$ is the annealing time, and $D$ is the mutual diffusion coefficient. Using eq 1, Figure $6 b$ shows the best fit (dashed red line) to the interfacial broadening. To determine $Z^{*}$, numerical integration between this fit, with the lowest reduced $x^{2}$, and the experimental data yield the surface excess denoted by the solid teal region in Figure $6 \mathrm{~b}$.

a.

As-Cast

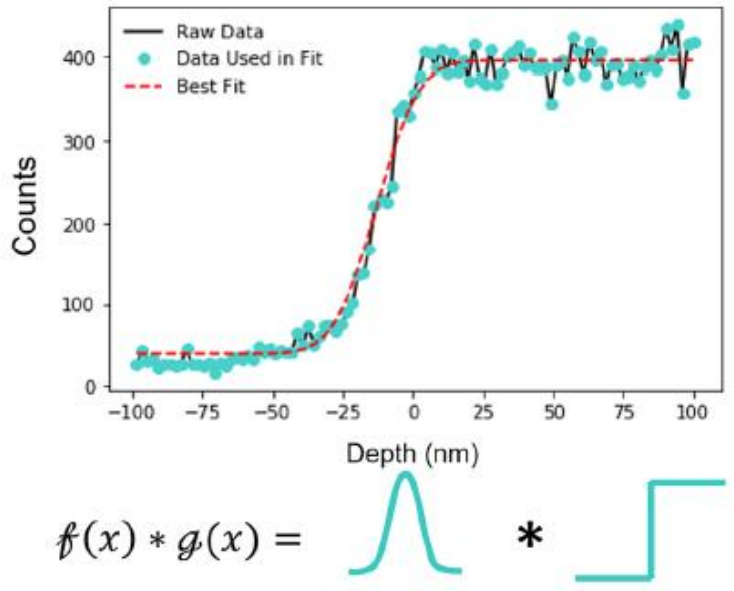

b.

$30 \mathrm{~min}, 170^{\circ} \mathrm{C}$
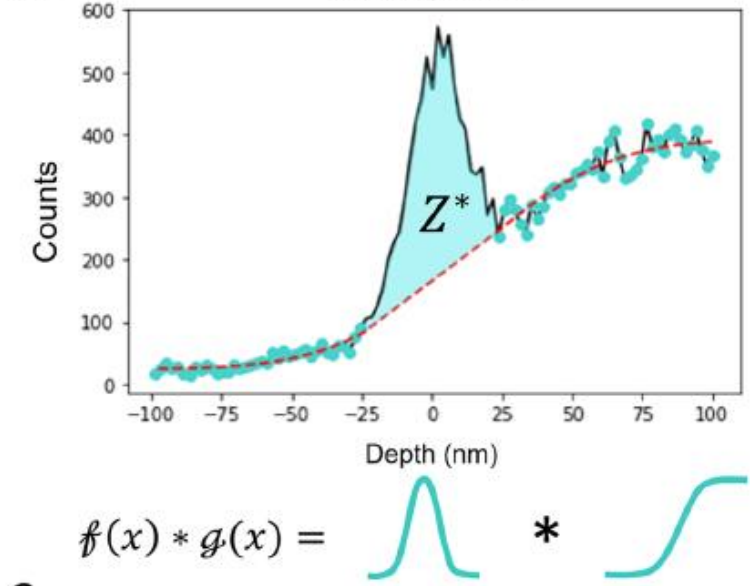

C.

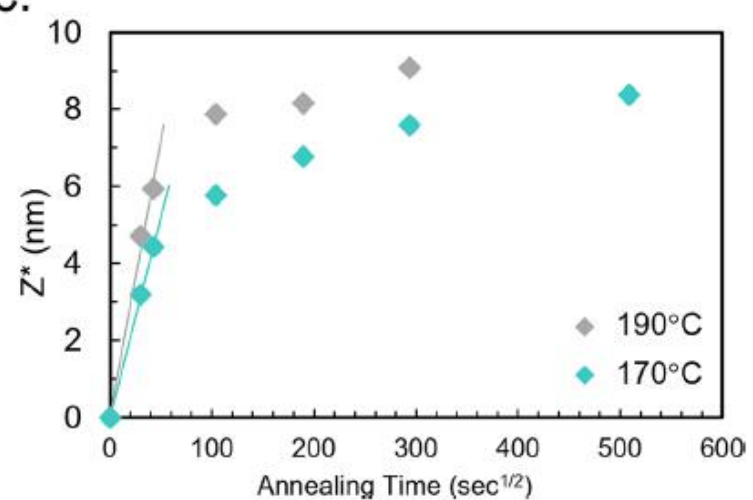

Figure 6. Depth profiles of PMMA-NPs in 25/75 wt \% PMMA-NP/ SAN films (a) before and (b) after annealing at $170{ }^{\circ} \mathrm{C}$ for 30 $\mathrm{min}$, as revealed by GI-RBS. The dashed red lines are the fits to the experimental data corresponding to (a) a Gaussian instrumental resolution convoluted with a step function and (b) a Gaussian instrumental resolution convoluted with a solution of Fick's second law given by eq 1 . The surface excess of PMMA-NP is the shaded area in (b), which is denoted as $Z^{\star}$. (c) Surface excess of PMMA-NP plotted as a function of the square root of the annealing time. The solid lines are the best fits of the slopes in the linear growth regime. 
The NP diffusion coefficients can be determined from the growth of the integrated surface excess. As originally pointed out by Jones and Kramer,20,21 conservation of mass requires that the excess material at the surface equals the amount removed from the depleted region. Therefore, sufficiently far from the surface, the width of the depletion zone should scale as (Dt $)^{1 / 2}$. The following approximation can be used:

$$
Z^{*}=\left(\varphi_{\text {Bulk }}-\varphi_{\text {Depletion }}\right)(D t)^{1 / 2}
$$

where $\varphi$ Depletion is the depletion region volume fraction determined from the profile, as shown in Figure $6 \mathrm{~b}$. Because of the high loading of PMMA-NP and enthalpic driving force, $D$ represents a mutual diffusion coefficient and can be calculated from the experimental $Z^{*}$ values plotted in Figure $6 \mathrm{c}$ versus $\mathrm{t}^{1 / 2}$. For both temperatures, $Z^{*}$ rapidly increases with time before approaching constant values. This plateau of $Z^{*}$ at long durations of time is attributed to a reduction in the driving force for NPs to segregate to the free surface and steric hindrance originating from NPs already occupying interfacial sites. At each time point, $Z^{*}$ at $190{ }^{\circ} \mathrm{C}$ is greater than that at $170^{\circ} \mathrm{C}$, consistent with faster diffusion at the higher temperature (and deeper quench depth). Taking an average value of $\varphi$ Depletion together with the best fit value for the slopes of the linear regime (solid lines), the growth of $Z^{*}$ at 170 and $190^{\circ} \mathrm{C}$ yield mutual diffusion coefficients of $4.3 \times 10^{-14} \mathrm{~cm}^{2} / \mathrm{s}$ and $6.4 \times 10^{-14} \mathrm{~cm}^{2} / \mathrm{s}$, respectively.

We compare the experimentally obtained diffusion coefficients to those calculated using the continuum-based Stokes - Einstein (SE) relation, which is a common benchmark for comparing particle diffusion in soft matter. Here, it is important to note that the SE model assumes a dilute NP concentration, whereas the experimental system contains $25 \mathrm{wt} \%$ PMMA-NP, which is above the dilute limit. Recently, Lin and co-workers performed RBS experiments to address the impact of grafted polymers on the diffusion of NP.37 Interestingly, their results showed that grafted polymer chains of moderate molecular weight and grafting density significantly alter NP diffusion in polymer melts by increasing their effective size. Therefore, because of the high grafting density and molecular weight of the brushes used in the present study, an effective NP radius of $R_{\text {eff }}=19 \mathrm{~nm}$ is used in the SE model. Using this effective radius and the viscosity of SAN, the PMMA-NP diffusion coefficients are $1.2 \times 10^{-16} \mathrm{~cm}^{2} / \mathrm{s}$ and $2.5 \times 10^{-15} \mathrm{~cm}^{2} / \mathrm{s}$ at 170 and $190{ }^{\circ} \mathrm{C}$, respectively. The SE predicted values in the dilute limit are significantly less than the experimental values, $D_{S E}<<z^{*}$. Because the viscosity of the $25 \mathrm{wt} \%$ PMMA-NP/SAN blend should be even greater than that of pure SAN, DSE is less than this prediction. It is worth noting that SE predicts a much stronger temperature dependence than that observed in the experimental results. Specifically, whereas $\operatorname{DSE}\left(190^{\circ} \mathrm{C}\right) / \mathrm{DSE}_{\mathrm{SE}}\left(170^{\circ} \mathrm{C}\right) \approx 4.8$, the ratio is about three times smaller for the experimentally measured values, $\mathrm{Dz}_{z^{*}}\left(190^{\circ} \mathrm{C}\right) / \mathrm{Dz}^{*}\left(170^{\circ} \mathrm{C}\right) \approx 1.5$. This observation indicates that the temperature dependence of $D$ is much weaker than that expected from changes in viscosity alone.

To investigate the role of the NP size in enhanced diffusion, Schweizer and co-workers developed a microscopic, force-level, self-consistent generalized Langevin equation (SCGLE) approach to predict the diffusion coefficient of repulsive or athermal NPs as a function of particle size and molecular weight of the matrix.38 Using SCGLE, NP mobility is found to exhibit size-dependent, non-SE behavior that falls in two regimes: (1) a fast diffusion regime that depends on the relaxation of the unentangled matrix chains when $2 R_{\text {eff }}$ is less than the tube diameter of the matrix (dt) and (2) a much slower diffusion regime dependent on polymer relaxations via reptation when the particle size is greater than dt. The transition between these two regimes is relatively sharp, and the SE NP diffusion is recovered when $2 R_{\text {eff }} / \mathrm{dt} \sim 10.38$ Using the Kuhn length and Kuhn monomer molar mass of polystyrene as an approximation for SAN, the tube diameter is ca. $9 \mathrm{~nm}$. Based on the results presented by Schweizer and co-workers, a 2Reff/dt ratio of $\sim 4$ should lead to an enhancement in the diffusion coefficient relative to $S E$ by $D / D_{S E} \approx 3.6$. For comparison, if we calculate $\mathrm{D} / \mathrm{D}_{\mathrm{SE}}$ using the experimental $\left(\mathrm{D}_{Z^{*}}\right)$ and theoretical $\left(\mathrm{D}_{\mathrm{SE}}\right)$ values for $\mathrm{D}$, we see that $\mathrm{D}_{Z^{*}} / \mathrm{D}_{\mathrm{SE}}\left(170^{\circ} \mathrm{C}\right) \approx 360$ and $\mathrm{Dz}^{*} / \mathrm{DSE}_{\mathrm{SE}}\left(190^{\circ} \mathrm{C}\right) \approx 26$, both of which are orders of magnitude higher than those predicted using the SCGLE model.

It is important to reiterate that the SE relationship is purely entropic in origin and describes the competition between the thermal fluctuations of the system and viscous drag forces of the medium. Additionally, the theoretical predictions made by Schweizer and co-workers are for a thermal and weakly interacting PNCs. Although these approaches assume hard spheres, recent molecular dynamic simulations capture the effect of polymer tethers on NP diffusion through unentangled polymer matrices.39 As a result, the large discrepancy between the theoretical diffusion coefficients and those obtained experimentally comes at no surprise. A complete model for the present study would include driving forces because of both wetting and phase separation, which will significantly influence NP diffusion. Therefore, we hope that this study demonstrates the enhancement of NP diffusion toward surfaces upon phase separation in a PNC and motivates the development of theory and simulations that capture the diffusion during simultaneous wetting and phase separation in the two-phase region.

Because the dynamics of PNCs are critically important to understand in the design and fabrication of functional PNCs, studies such as those discussed herein are essential.40 For instance, the ability to reliably model processes such as the surface enrichment in a mixed condensed phase requires accurate knowledge of NP diffusion that simple models, for example, SE, fail to accurately capture because of the complex phase behavior described in the present study. Riggleman et al. have quite recently extended the equilibrium PNC field theory (PNC-FT) to capture the kinetics of self-assembly in NP composites.41-43 These theoretically informed Langevin dynamics (TILD) simulations allow one to efficiently predict kinetic pathways during the self-assembly of PNCs.44,45 As a result, the TILD calculations can potentially predict PNC morphologies using accurate thermodynamic and kinetic inputs. Importantly, for certain processes, the TILD calculations would better capture experimental behavior if accurate diffusion coefficients are used rather than the traditional estimates based on diffusion models such as SE. Overall, the thermodynamic and 
kinetic intuition gained from this study offers a facile route for preparing PNC structures with the potential for enhanced surface-dependent properties.

\section{CONCLUSIONS}

We have measured the wetting behavior and internal morphology in a PNC of PMMA-NPs and SAN. By annealing deep and shallow in the two-phase region, the thermodynamic and kinetic factors controlling phase separation have been investigated. The wetting and phase-separation stages in the PNC are similar to those observed for pure polymer blends where the lower surface energy component (PMMA-NPs) rapidly wets the free surface to reduce the total free energy. The growth of the NP surface excess is measured, and it provides an alternative approach to determine the mutual diffusion coefficient at high loadings of NPs. The development of a unique nanocomposite morphology is revealed because of the preferential wetting during phase evolution. Specifically, PMMA-NPs form elongated aggregates or discrete columns that span the thickness of the film. Additionally, the morphology exhibits PMMA-NP wetting layers separated by a continuous SAN-rich matrix perforated with the NP columns. This phase structure has great potential as a facile route for preparing nanocomposite films with increased mechanical properties for barrier coatings, analogous to the carburization of steel, or other surface-dependent properties. More specifically, the mechanical properties of the nanocomposites should be improved, relative to neat polymer blends, because the jammed NP wetting layers and columns resist deformation, similar to what has been demonstrated through the suppression of interfacial fluctuations upon heating. A detailed comparison of film stability and the mechanical properties (elastic modulus and hardness) between the polymer blend and PNC will be examined in future studies.

\section{METHODS}

Materials Characterization. SAN $\left(\mathrm{Mw}=118 \mathrm{~kg} / \mathrm{mol}, \mathrm{M}_{\mathrm{w}} / \mathrm{M}_{\mathrm{n}}=2.24, \mathrm{~T}_{\mathrm{g}}=114{ }^{\circ} \mathrm{C}\right.$, containing $33 \mathrm{wt} \%$ acrylonitrile) was provided by Monsanto and purified twice by adding a solution of SAN and chloroform ( $\geq 99.9 \%$, for HPLC) into methanol $(\geq 99.9 \%$, for HPLC) at a 1:10 volume ratio. After allowing SAN to precipitate for $1 \mathrm{~h}$, the solvent was removed, and the precipitant was dried for $48 \mathrm{~h}$. Once dried, the SAN was redissolved in chloroform and precipitated with methanol once more. PMMA $\left(\mathrm{Mw}=21 \mathrm{~kg} / \mathrm{mol}, \mathrm{M}_{\mathrm{w}} / \mathrm{M}_{\mathrm{n}}=1.14, \mathrm{~T}_{\mathrm{g}}=110^{\circ} \mathrm{C}\right)$ was purchased from Polymer Source Inc. and used as received. Silica nanoparticles (15 nm diameter) grafted with PMMA brushes, denoted as PMMANPs, were prepared using surface-initiated atom transfer radical polymerization. The brush weight average molecular weight and grafting density were $19 \mathrm{~kg} / \mathrm{mol}$ and 0.7 chains $/ \mathrm{nm}^{2}$, respectively, as determined by thermogravimetric analysis (TGA, TA Instruments Q600 SDT) at a heating rate of $10^{\circ} \mathrm{C} / \mathrm{min}$ under argon flow between the temperatures of 25 and $550{ }^{\circ} \mathrm{C}$. The glass transition temperature $\left(T_{g}\right)$ of each polymer was measured using a differential scanning calorimeter (TA Instruments Q2000) between the temperatures of 25 and $150^{\circ} \mathrm{C}$. In all cases, two heating/cooling cycles were performed at a heating/cooling rate of $5{ }^{\circ} \mathrm{C} / \mathrm{min}$. The $T_{g}$ values were obtained from the second heating in order to eliminate any thermal history. The hydrodynamic radius of the PMMA-NPs was determined to be $19 \mathrm{~nm}$ by dynamic light scattering (DLS, Malvern Zetasizer nano-s). Details of TGA, differential scanning calorimetry, and DLS characterization of PMMA, SAN and PMMA-NPs are given in the Supporting Information of our previous work.34 At all experimentally probed temperatures, the surface energy of the PMMA-NPs is less than that of the SAN matrix (see the Supporting Information). EpoxiCure ${ }^{2}$ epoxy hardener and epoxy resin were purchased from Buehler. N-type, $<100>$ oriented silicon wafers (dopant Ph, 10-20 $\Omega \cdot \mathrm{cm}$ resistivity, 475-575 $\mu \mathrm{m}$ thickness, single-side polished) were purchased from Silicon Quest International. P-type, $<100>$ oriented silicon wafers (dopant B, 0.001-0.005 $\Omega \cdot \mathrm{cm}$ resistivity, $500 \mu \mathrm{m}$ thickness, single side polished) with a $300 \mathrm{~nm}$ wet thermal oxide layer were purchased from University Wafer.

AFM. PNCs were imaged in the tapping mode using an Agilent 5420 AFM with noncontact tips (TAP300AL-G-50 radius of curvature $<10 \mathrm{~nm}$, Ted Pella) as a function of thermal annealing temperature and time. All AFM images were processed using the Gwyddion software. Radial autocorrelation functions of the $2 \mu \mathrm{m} \times 2 \mu \mathrm{m}$ images were produced to discern the average center-to-center interparticle distances (IDs) of the grafted NPs at the film surface. The surface coverage of the grafted NPs as a function of annealing temperature and time was extracted using standard image processing techniques in the ImageJ software (see Supporting Information).

Optical Microscopy. To compare the dewetting behavior of the PMMA/SAN blend and PMMA-NP/SAN PNC, solutions were prepared by dissolving the constituents in methyl isobutyl ketone (MIBK) and stirred for $24 \mathrm{~h}$ using a magnetic stir bar. Both solutions (10 wt \% in MIBK) were spin-coated (4000 rpm, $60 \mathrm{~s})$ onto $1 \mathrm{~cm} \times 1 \mathrm{~cm}$ silicon wafers with no thermal oxide layer and then dried using the aforementioned procedure. After thermal annealing at $190{ }^{\circ} \mathrm{C}$ for varying amounts of time, the samples were examined by OM (Olympus BH-2) in reflected light configuration using a $10 \times$ objective lens and $1.67 \times$ relay lens.

TEM. PNC solutions were prepared by dissolving the constituents in MIBK ( $\geq 98.5 \%$, Certified ACS, Fisher Chemical) and stirred for $24 \mathrm{~h}$ using a magnetic stir bar. The solutions (10 wt \% in MIBK) were spin-coated (4000 rpm, $60 \mathrm{~s}$ ) onto $1 \mathrm{~cm} \times 1 \mathrm{~cm}$ silicon wafers with a $300 \mathrm{~nm}$ thermal oxide layer and then dried at $100{ }^{\circ} \mathrm{C}$ for $1 \mathrm{~h}$ to remove the residual solvent. These conditions yield $400 \mathrm{~nm}$ films, suitable for capturing the entire film cross-section in a single TEM image. Following drying, the samples were annealed on a hot stage at 170 and $190^{\circ} \mathrm{C}$ for varying amounts of time under continuous argon flow and then immediately quenched to room temperature (RT). To lift the films, edges of the substrates were scored and then floated on a $1: 5$ vol \% solution of $\mathrm{NaOH}(50 \% \mathrm{w} / \mathrm{w} \mathrm{NaOH})$ and $\mathrm{DI} \mathrm{H} \mathrm{H}_{2} \mathrm{O}$. After lifting from the substrates, films were transferred from the liquid-air interface of the $\mathrm{NaOH}$ solution to $\mathrm{DI}_{2} \mathrm{H}_{2} \mathrm{O}$ and then onto Teflon. The specimens were then sandwiched between two pieces of tape, with an open window on one side to expose the free surface of the PNC (seethe Supporting Information). Next, the samples were embedded in two-part epoxy. Once the epoxy cured, $\sim 70-100 \mathrm{~nm}$ cross-sections of the PNCs were prepared by ultramicrotomy 
(Leica Ultracut S Ultramicrotome), with a RT diamond knife. TEM characterization of the ultramicrotomed crosssections, on carbon-coated TEM grids, was performed with a JEOL JEM-1400 TEM operated at $120 \mathrm{kV}$.

GI-RBS. To prepare the samples for GI-RBS, the same PNC solutions used for TEM were spin-coated (4000 rpm, $60 \mathrm{~s}$ ) onto $1 \mathrm{~cm} \times 1 \mathrm{~cm}$ silicon wafers with no thermal oxide layer and then dried using the aforementioned procedure. After thermal annealing at 170 and $190^{\circ} \mathrm{C}$ for varying amounts of time, the $400 \mathrm{~nm}$ thick samples were examined by GI-RBS using an incident beam of 2-MeV He+ ions produced using a National Electrostatics 5SDH-2 positive-ion accelerator. The GI-RBS geometry consisted of an incident ion beam of $60^{\circ}$ off the surface normal, and an exit angle of $65^{\circ}$, resulting in a $175^{\circ}$ backscatter. After obtaining the GI-RBS spectra, the counts versus channel profiles were translated into counts versus depth using an energy calibration of $1.709 \mathrm{keV} / \mathrm{channel}$ with an offset of $+95 \mathrm{keV}$, obtained from a $10 \mathrm{~nm}$ AuPd calibration sample. Because the PNC films are orders of magnitude thinner than the Si wafer, substrates were modeled as infinite slabs. Each GI-RBS spectrum was normalized to the same silicon shelf to mitigate differences in collection times.

\section{ASSOCIATED CONTENT}

\section{SI: Supporting Information}

The Supporting Information is available free of charge at https://pubs.acs.org/doi/10.1021/acsami.1c09233. Constituent surface energies, AFM image analysis, sample preparation, experimental methods, and additional figures (PDF)

\section{AUTHOR INFORMATION \\ Corresponding Author}

Russell J. Composto - Department of Materials Science and Engineering, University of Pennsylvania, Philadelphia, Pennsylvania 19104, United States; orcid.org/0000-0002-5906-2594; Email: composto@seas.upenn.edu

\section{Authors}

Shawn M. Maguire - Department of Materials Science and Engineering, University of Pennsylvania, Philadelphia, Pennsylvania 19104, United States; orcid.org/0000- 0002-5317-4990

Michael J. Boyle - Department of Materials Science and Engineering, University of Pennsylvania, Philadelphia, Pennsylvania 19104, United States

Connor R. Bilchak - Department of Materials Science and Engineering, University of Pennsylvania, Philadelphia, Pennsylvania 19104, United States; orcid.org/0000-0001-8815-1601

John Derek Demaree - US Army Research Laboratory, Aberdeen Proving Ground, Maryland 21005, United States Austin W. Keller - Department of Materials Science and Engineering, University of Pennsylvania, Philadelphia,Pennsylvania 19104, United States

Nadia M. Krook - Department of Materials Science and Engineering, University of Pennsylvania, Philadelphia, Pennsylvania 19104, United States; Present Address: DuPont Co., Wilmington, Delaware 19,803, United States

Kohji Ohno - Institute for Chemical Research, KyotoUniversity, Kyoto 611-0011, Japan; orcid.org/0000-0002-18123354

Cherie R. Kagan - Department of Materials Science and Engineering, Department of Chemistry, and Department of Electrical and Systems Engineering, University of Pennsylvania, Philadelphia, Pennsylvania 19104, United States; orcid.org/0000-0001-6540-2009

Christopher B. Murray - Department of Materials Science and Engineering and Department of Chemistry, University of Pennsylvania, Philadelphia, Pennsylvania 19104, United States

Patrice Rannou - Univ. Grenoble Alpes, CNRS, CEA, IRIGSyMMES,38000 Grenoble, France; orcid.org/0000-00019376-7136

Complete contact information is available at: https://pubs.acs.org/10.1021/acsami.1c09233

\section{Author Contributions}

R.J.C. designed and supervised this work. S.M.M., M.J.B., C.R.B., J.D.D., A.W.K., and N.M.K. carried out experiments. S.M.M., M.J.B., and J.D.D. analyzed experimental results. S.M.M. prepared schematics. The manuscript was written and edited through contributions of all authors. All authors have given approval to the final version of the manuscript.

\section{Notes}

The authors declare no competing financial interest.

\section{ACKNOWLEDGMENTS}

S.M.M., C.R.B., and R.J.C. were supported by the National Science Foundation Partnerships for International Research and Education Program (NSF-PIRE), Grant \#1545884. This work was additionally supported by the POLYMERS DMR1905912 (S.M.M., N.M.K., C.R.B., and R.J.C.) and MRSEC-DMR-1720530 (S.M.M., N.M.K., C.R.B., and R.J.C.) programs. S.M.M. and P.R. also acknowledge support from the Centre National de la Recherche Scientifique (CNRS) at the laboratory of "Systèmes Moléculaires et nanoMatériaux pour l'Energie et la Santé," (UMR5819-SyMMES (CNRS/CEA/Univ. Grenoble Alpes), Grenoble, France) and funding from the Agence Nationale de la Recherche (ANR): ANR-15-PIRE-0001-01 and ANR-15-PIRE-0001-07. M.J.B. acknowledges funding from an NSF Graduate Fellowship as well as NSFCBET-1706014 (R.J.C. and M.J.B.). A.W.K., C.R.K., and C.B.M. acknowledge funding from the Semiconductor Research Corporation (SRC), Task 2797.001. We thank Dr.Karen I. 
Winey and Dr. Zahra Fakhraai for use of their laboratory's ultramicrotome and AFM, respectively. The authors also acknowledge Dr. Robert A. Riggleman, Dr. Daeyeon Lee, and Dr. James F. Pressly for helpful discussion

\section{REFERENCES}

(1) Green, P. F. The Structure of Chain End-Grafted Nanoparticle/ Homopolymer Nanocomposites. Soft Matter 2011, 7, 79147926.

(2) Winey, K. I.; Vaia, R. A. Polymer Nanocomposites. MRS Bull. 2007, 32, 314-322.

(3) Kumar, S. K.; Ganesan, V.; Riggleman, R. A.; Kumar, S. K.; Ganesan, V.; Riggleman, R. A. Perspective: Outstanding Theoretical Questions in Polymer-Nanoparticle Hybrids. J. Chem. Phys. 2017, 147, No. 020901.

(4) Choi, J.; Dong, H.; Matyjaszewski, K.; Bockstaller, M. R. Flexible Particle Array Structures by Controlling Polymer Graft Architecture. J. Am. Chem. Soc. 2010, 132, 12537-12539.

(5) Choi, J.; Hui, C. M.; Pietrasik, J.; Dong, H.; Matyjaszewski, K.; Bockstaller, M. R. Toughening Fragile Matter: Mechanical Properties of Particle Solids Assembled from Polymer-Grafted Hybrid Particles Synthesized by ATRP. Soft Matter 2012, 8, 4072 $-4082$.

(6) Gao, J.; Li, J.; Benicewicz, B. C.; Zhao, S.; Hillborg, H.; Schadler, L. S. The Mechanical Properties of Epoxy Composites Filled with Rubbery Copolymer Grafted SiO2. Polymers (Basel). 2012, 4, 187-210.

(7) Buitrago, C. F.; Pressly, J. F.; Yang, A. S.; Gordon, P. A.; Riggleman, R. A.; Natarajan, B.; Winey, K. I. Creep Attenuation in Glassy Polymer Nanocomposites with Variable Polymer-Nanoparticle Interactions. Soft Matter 2020, 16, 8912-8924.

(8) Naskar, A. K.; Keum, J. K.; Boeman, R. G. Polymer Matrix Nanocomposites for Automotive Structural Components. Nat. Nanotechnol. 2016, 11, 1026-1030.

(9) Chai, S.; Cao, X.; Xu, F.; Zhai, L.; Qian, H.; Chen, Q.; Wu, L.; Li, H. Multiscale Self-Assembly of Mobile-Ligand Molecular Nanoparticles for Hierarchical Nanocomposites. ACS Nano 2019, 13,

7135-7145.

(10) Huang, Y.; Jiang, Y.; Hor, J. L.; Gupta, R.; Zhang, L.; Stebe, K. J.; Feng, G.; Turner, K. T.; Lee, D. Polymer Nanocomposite Films with Extremely High Nanoparticle Loadings via Capillary Rise Infiltration (CaRI). Nanoscale 2015, 7, 798-805.

(11) Wang, H.; Hor, J. L.; Zhang, Y.; Liu, T.; Lee, D.; Fakhraai, Z. Dramatic Increase in Polymer Glass Transition Temperature under Extreme Nanoconfinement in Weakly Interacting Nanoparticle Films.

ACS Nano 2018, 12, 5580-5587.

(12) Wang, H.; Qiang, Y.; Shamsabadi, A. A.; Mazumder, P.; Turner, K. T.; Lee, D.; Fakhraai, Z. Thermal Degradation of Polystyrene under Extreme Nanoconfinement. ACS Macro Lett. 2019, 8, 1413-1418.

(13) Ginzburg, V. V. Influence of Nanoparticles on Miscibility of Polymer Blends. A Simple Theory. Macromolecules 2005, 38, 2362-2367.

(14) Ferrier, R. C.; Koski, J.; Riggleman, R. A.; Composto, R. J. Engineering the Assembly of Gold Nanorods in Polymer Matrices. Macromolecules 2016, 49, 1002-1015.

(15) Krook, N. M.; Ford, J.; Marechal, M.; Rannou, P.; Meth, J. S.; Murray, C. B.; Composto, R. J. Alignment of Nanoplates in Lamellar-Forming Diblock Copolymer Domains. ACS Macro Lett. 2018, 7, 1400-1407.

(16) Rasin, B.; Chao, H.; Jiang, G.; Wang, D. Soft Matter Dispersion and Alignment of Nanorods in Cylindrical Block Copolymer Thin Films. Soft Matter 2016, 12, 2177-2185.

(17) Glynos, E.; Petropoulou, P.; Mygiakis, E.; Nega, A. D.; Pan, W.; Papoutsakis, L.; Giannelis, E. P.; Sakellariou, G.; Anastasiadis, S. H. Leveraging Molecular Architecture To Design New, All-Polymer Solid Electrolytes with Simultaneous Enhancement in Modulus and lonic Conductivity. Macromolecules 2018, 51, 2542-2550.

(18) Shull, K. R.; Winey, K. I.; Thomas, E. L.; Kramer, E. J. Segregation of Block Copolymer Micelles to Surfaces and Interfaces. Macromolecules 1991, 24, 2748-2751.

(19) Jones, R. A. L.; Kramer, E. J. The Surface Composition of Miscible Polymer Blends. Polymer 1993, 34, 115-118.

(20) Zhao, X.; Zhao, W.; Sokolov, J.; Rafailovich, M. H.; Schwarz, S. A.; Wilkens, B. J.; Jones, R. A. L.; Kramer, E. J. Determination of the Concentration Profile at the Surface of D-PS/h-PS Blends Using High-Resolution Ion Scattering Techniques. Macromolecules 1991, 24, 5991-5996.

(21) Jones, R. A. L.; Kramer, E. J.; Rafailovich, M. H.; Sokolov, J.; Schwarz, S. A. Surface Enrichment in an Isotopic Polymer Blend. Phys. Rev. Lett. 1989, 62, 280-283.

(22) Binder, K. Phase Transitions of Polymer Blends and Block Copolymer Melts in Thin Films. In Polymers in Confined Environments Granick, S., Binder, K., de Gennes, P.G., Giannelis, E.P., Grest, G.S., Hervert, H., Krishnamoorti, R., Léger, L., Manias, E., Raphaël, E., Wang, S.Q. Eds., Springer: Berlin, Heidelber, 1999, 138.

(23) Chung, H.-J.; Kim, J.; Ohno, K.; Composto, R. J. Controlling the Location of Nanoparticles in Polymer Blends by Tuning the Length and End Group of Polymer Brushes. ACS Macro Lett. 2012, 1, 252-256.

(24) Chung, H.-J.; Ohno, K.; Fukuda, T.; Composto, R. J. Internal Phase Separation Drives Dewetting in Polymer Blend and Nanocomposite Films. Macromolecules 2007, 40, 384-388.

(25) Chung, H.-J.; Ohno, K.; Fukuda, T.; Composto, R. J. Self- Regulated Structures in Nanocomposites by Directed Nanoparticle Assembly. Nano Lett. 2005, 5, 1878-1882.

(26) Gam, S.; Corlu, A.; Chung, H.-J.; Ohno, K.; Hore, M. J. A.; Composto, R. J. A Jamming Morphology Map of Polymer Blend Nanocomposite Films. Soft Matter 2011, 7, 7262-7268.

(27) Chung, H.-J.; Wang, H.; Composto, R. J. A Morphology Map Based on Phase Evolution in Polymer Blend Films. Macromolecules 2006, 39, 153-161.

(28) Maguire, S. M.; Chung, H.-J.; Composto, R. J. Polymer Blend Systems With an Added Solvent. In Bijels: Bicontinuous Particlenstabilized Emulsions; Clegg, P. S., Ed.; Royal Society of Chemistry, 2020; pp. 73-113.

(29) Wang, H.; Composto, R. J. Thin Film Polymer Blends Undergoing Phase Separation and Wetting : Identification of Early,Intermediate, and Late Stages. J. Chem. Phys. 2000, 113, 10386.

(30) Chung, H.-J.; Kim, J.; Ohno, K.; Composto, R. J. Controlling the Location of Nanoparticles in Polymer Blends by Tuning the Length and End Group of Polymer Brushes. ACS Macro Lett. 2012, 1, 252-256.

(31) Chung, H.-J. Phase Behavior of Confined Polymer Blends and Nanoparticle Composites, University of Pennsylvania, 2005. 
(32) Chung, H.-J.; Ohno, K.; Fukuda, T.; Composto, R. J. Self-Regulated Structures in Nanocomposites by Directed Nanoparticle Assembly. Nano Lett. 2005, 5, 1878-1882.

(33) Newby, B. Z.; Composto, R. J. Influence of Lateral Confinement on Phase Separation in Thin Film Polymer Blends. Macromolecules 2000, 33, 3274-3282.

(34) Maguire, S. M.; Krook, N. M.; Kulshreshtha, A.; Bilchak, C. R.; Brosnan, R.; Pana, A. M.; Rannou, P.; Maréchal, M.; Ohno, K.; Jayaraman, A.; Composto, R. J. Interfacial Compatibilization in Ternary Polymer Nanocomposites: Comparing Theory and Experiments.Macromolecules 2021, 54, 797-811.

(35) Wang, H.; Composto, R. J. Hydrodynamic-Flow-Driven Wetting in Thin Film Polymer Blends : Growth Kinetics and Morphology. Phys. Rev. E 2000, 61, 1659-1663.

(36) Wang, H.; Composto, R. J. Kinetics of Surface and Interfacial Fluctuations in Phase Separating Polymer Blend Films. Macromolecules 2002, 35, 2799-2809.

(37) Lin, C. C.; Griffin, P. J.; Chao, H.; Hore, M. J. A.; Ohno, K.; Clarke, N.; Riggleman, R. A.; Winey, K. I.; Composto, R. J. Grafted Polymer Chains Suppress Nanoparticle Diffusion in Athermal Polymer Melts. J. Chem. Phys. 2017, 146, 203332.

(38) Yamamoto, U.; Schweizer, K. S. Microscopic Theory of the Long-Time Diffusivity and Intermediate-Time Anomalous Transport of a Nanoparticle in Polymer Melts. Macromolecules 2015, 48, 152-163.

(39) Ge, T.; Rubinstein, M.; Grest, G. S. Effects of TetheredPolymers on Dynamics of Nanoparticles in Unentangled Polymer Melts. Macromolecules 2020, 53, 6898-6906.

(40) Bailey, E. J.; Winey, K. I. Dynamics of Polymer Segments, Polymer Chains, and Nanoparticles in Polymer Nanocomposite Melts: A Review. Prog. Polym. Sci. 2020, 105, No. 101242.

(41) Chao, H.; Hagberg, B. A.; Riggleman, R. A. The Distribution of Homogeneously Grafted Nanoparticles in Polymer Thin Films and Blends. Soft Matter 2014, 10, 8083-8094.

(42) Koski, J.; Chao, H.; Riggleman, R. A. Field Theoretic Simulations of Polymer Nanocomposites. J. Chem. Phys. 2013, 139, 244911.

(43) Koski, J.; Chao, H.; Riggleman, R. A. Predicting the Structure and Interfacial Activity of Diblock Brush, Mixed Brush, and Janus- Grafted Nanoparticles. Chem. Commun. 2015, 51, 5440-5443.

(44) Chao, H.; Koski, J.; Riggleman, R. A. Solvent Vapor Annealing in Block Copolymer Nanocomposite Films: A Dynamic Mean Field Approach. Soft Matter 2017, 13, 239-249.

(45) Koski, J. P.; Ferrier, R. C.; Krook, N. M.; Chao, H.; Composto, R. J.; Frischknecht, A. L.; Riggleman, R. A. Comparison of Field-Theoretic Approaches in Predicting Polymer Nanocomposite Phase Behavior. Macromolecules 2017, 50, 8797-8809. 